\title{
L'insegnamento della letteratura italiana nei licei della Svizzera tedesca
}

\author{
Sara AlloATti \\ Institut für Erziehungswissenschaft \\ Università di Zurigo
}

\begin{abstract}
Gli approcci seguiti nell'insegnamento della letteratura italiana nei licei della Svizzera tedesca si riflettono nell'eterogeneità delle proposte didattiche dei manuali prodotti sul territorio e degli scenari e dossier condivisi sulla piattaforma collaborativa italianoascuola.ch. In alcuni, si può notare una tendenza a un approccio ermeneutico che ben si concilia con la scelta prevalente di trattare approfonditamente poche letture integrali, ma non restano isolati canoni orientati a criteri storico-biografici e perlomeno parziali trattazioni di testi in forma antologica. Anche se mancano dati rappresentativi sulle tipologie di testi letti e di attività didattiche svolte, sono diverse le fonti attraverso le quali è possibile rilevare perlomeno una selezione dei testi più letti.
\end{abstract}

Keywords: letteratura di lingua italiana, insegnamento dell'italiano LS, manualistica, reti tra insegnanti, piattaforma partecipativa.

"Stare al passo col programma", questa era l'ansia di un mio professore di italiano - perché all'esame di maturità nazionale italiano ogni lacuna poteva essere fatale, diceva. E così, per cinque anni di liceo, e ogni anno in compagnia di una spessa antologia, scoprivamo a estratti, secolo dopo secolo, la storia della letteratura italiana. Solo saltuariamente leggevamo un testo integrale, e solo se il tempo lo permetteva: la trattazione antologica prevaleva ancor più marcatamente nelle lingue straniere, dove estratti di Chaucer e Shakespeare in lingua originale erano corredati da parafrasi e paratesti. Così ricordo le lezioni di letteratura di trent'anni fa in un liceo italiano - se ancora oggi sia così, non saprei dirlo.

So però che, iscrittami all'università in Svizzera tedesca, ebbi l'impressione che i proseminari di letteratura non fossero poi così diversi dalle quelle lezioni al liceo. Per l'analisi del testo si faceva ricorso a strumenti simili - e in assenza del volumone antologico, senza le sue schede di analisi e gli estratti di critica letteraria, l'analisi del testo era diventata una nuova sfida. Lo scarto tra liceo e università, per me, non è stato poi così notevole. Lo era invece soprattutto per compagne e compagni che provenivano da licei della Svizzera tedesca. L'uso rigoroso di determinati metodi d'analisi risultava a loro nuovo, parimenti alla terminologia ad essi connessa. Capii tuttavia ben presto, nei seminari, che loro avevano una marcia in più quando si trattava di penetrare un testo in profondità, di farlo risuonare nella propria cassa toracica per capirne i messaggi, per interpretarlo anche alla luce delle associazioni e delle emozioni che in loro generava. 
A me, infatti, mancava l'abitudine a svolgere attività "intorno al testo" come: "Hai già sperimentato un sentimento analogo a quello che descrive Leopardi nell'Infinito?" o "Immagina di essere un/a Dante dei giorni nostri e definisci una struttura dell'Inferno che rispecchi la morale della società dei nostri tempi.", o ancora "Ti sei mai sentito/a come Novecento, incapace di abbandonare la tua nave?". Attività di questo tipo prevedono di abbandonare i soli livelli di comprensione e analisi di un testo, presuppongono un allontanamento dal testo ma preparano un ritorno ad esso, con l'obiettivo di favorire uno sguardo "complice" e predisposto all'ascolto del testo stesso. Si tratta di attività che richiedono tempo e che si possono difficilmente svolgere ripercorrendo per soli estratti secoli di letteratura. Per alcuni questo approccio al testo può risultare quasi blasfemo. Inizialmente, lo era anche per me: ero abituata a focalizzare la mia attenzione sull'oggetto di studio "testo", per esempio analizzando rapporti intratestuali e figure retoriche; per me il lettore era soggetto, e non oggetto dell'analisi. Farlo diventare oggetto coincideva con perdere obiettività e rigore nel processo di analisi - finché ho scoperto, nel corso della mia abilitazione all'insegnamento e in particolare negli stage nelle scuole, che queste strategie didattiche possono invece far leva sull'incredibile potenziale della letteratura come "scuola di empatia" e coinvolgere anche chi, senza diventare oggetto della riflessione e dell'analisi, quell'empatia non saprebbe svilupparla. E non da ultimo: ho potuto constatare che questo approccio, in Svizzera tedesca, è fortemente ancorato anche nell'insegnamento della Li.

Se consultiamo l'Ordinanza sulla maturità (ORM, Art. 5, Obiettivi degli studi) ${ }^{1}$ e il Piano quadro degli studi per le scuole superiori di maturità2 del 1994 notiamo che

I Nell'articolo 5 dell'ordinanza concernente il riconoscimento degli attestati liceali di maturità (ORM) del Consiglio federale (I995, 15.02.1995) si legge: «I. Lo scopo delle scuole che preparano alla maturità è quello di offrire alle proprie allieve e ai propri allievi, nella prospettiva di una formazione permanente, la possibilità di acquisire solide conoscenze di base, adatte al livello secondario, e favorire la formazione di uno spirito d'apertura e di un giudizio indipendente. Queste scuole non aspirano a conferire una formazione specialistica o professionale, bensì privilegiano una formazione ampia, equilibrata e coerente che dia alle allieve $\mathrm{e}$ agli allievi la maturità necessaria per intraprendere studi superiori e per svolgere nella società tutte quelle attività complesse che essa richiede. Esse sviluppano contemporaneamente l'intelligenza, la volontà, la sensibilità etica ed estetica come pure le attitudini fisiche delle loro allieve e dei loro allievi. 2. Le maturande e i maturandi devono essere capaci di acquisire un nuovo sapere, di sviluppare la curiosità, l'immaginazione, la facoltà di comunicazione, come pure di lavorare da soli e in gruppo. Essi esercitano il ragionamento logico e l'astrazione, ma anche il pensiero intuitivo, analogico e contestuale. Imparano così a familiarizzarsi con la metodologia scientifica. 3. Le maturande e i maturandi devono padroneggiare una lingua nazionale ed aver acquisito buone conoscenze di altre lingue nazionali o straniere. Essi devono essere capaci di esprimersi con chiarezza, precisione e sensibilità e imparare a scoprire le ricchezze e le particolarità delle culture di cui ogni lingua è il vettore. 4. Le maturande e i maturandi devono sapersi situare nel mondo naturale, tecnico, sociale e culturale nel quale vivono, nelle sue dimensioni svizzere e internazionali, attuali e storiche. Essi si preparano ad esercitarvi la loro responsabilità verso sé stessi, gli altri, la società e la natura».

2 Si considera qui la versione in lingua tedesca Rahmenlehrplan für die Maturitätsschulen vom 
mancano indicazioni su determinati programmi da seguire o su specifici temi da trattare. Entrambi i documenti descrivono competenze e attitudini che l'insegnamento liceale deve sviluppare, ma senza specificare i contenuti attraverso i quali tali obiettivi vadano raggiunti. I singoli cantoni possono, ma non devono, declinare queste linee guida in programmi di studio più dettagliati. Se non lo fanno, sono le singole scuole a poter (e non dover) compilare un proprio piano di studi. Nessuna pressione a seguire un programma, quindi, ma molteplici strade possibili per sviluppare "l'intelligenza, la volontà, la sensibilità etica ed estetica" dei e delle liceali. Chi insegna nei licei della Svizzera tedesca è perlopiù molto orgoglioso della propria "Lehrfreiheit" (libertà di insegnamento) e sostiene la tesi che quanto più l'insegnante si identifica con i contenuti che sceglie, tanto meglio riuscirà a raggiungere gli obiettivi formativi dell'ORM. La "Lehrfreiheit" si riflette così nella prassi: accanto, per esempio, a insegnanti che trattano solo testi moderni, altri propongono la lettura dei classici. Sono invece pochi coloro che mirano all'acquisizione di nozioni complete di "storia della letteratura"3: i più preferiscono letture integrali originali o con note di vocabolario da svolgersi a casa o in classe sull'arco di più settimane e accompagnano le letture con un vasto spettro di attività sul e intorno al testo, per permettere ad allievi e allieve di "acquisire un nuovo sapere, di sviluppare la curiosità, l'immaginazione, la facoltà di comunicazione, come pure di lavorare da soli e in gruppo" e di esercitare "il ragionamento logico e l'astrazione, ma anche il pensiero intuitivo, analogico e contestuale" (cfr. nota I) - la trattazione antologica, laddove presente, generalmente è complementare alle letture integrali.

Quanto ai metodi e alle tecniche didattiche in voga, manca in Svizzera tedesca una teorizzazione di un preciso modello di riferimento - diversamente da quanto è successo nella Provincia di Bolzano, dove si è imposto l'approccio ermeneutico (Magnani 2009), un paradigma che, come si vedrà, si ritrova nelle sue idee principali anche in alcune pubblicazioni didattiche sulla letteratura elvetiche - in particolare nel suo privilegiare la qualità alla quantità e allo spazio dato all'apprendente per incontrare il testo e per chiedersi quale sia, soggettivamente, il suo significato.

\section{Letteratura nella manualistica svizzero-tedesca}

La differenziata prassi elvetica si riflette nel panorama delle pubblicazioni svizzero-tedesche di scenari e materiali didattici a contenuti letterari. Un caso a parte è rappresentato dal corso di italiano per principianti Sì, leggia-

9. Juni 1994, Schweizerische Konferenz der kantonalen Erziehungsdirektoren (EDK).

3 Pare che la tendenza a trascurare la storia della letteratura si sia accentuata negli ultimi anni - fatto che ha portato di riflesso, p. es. all'Università di Zurigo, alla creazione di nuovi corsi di storia della letteratura che colmino le lacune individuate nelle nuove generazioni di studenti e studentesse. 
mo! (Schärer Curetti 1994; fig. I): sull'onda del metodo dell'extensive reading, il Lehrmittelverlag di Zurigo pubblica nel 1994 un manuale in cui l'apprendimento delle strutture linguistiche si vuole sostenuto dalla lettura di un grandissimo numero di testi autentici, tra cui filastrocche e racconti spesso di natura letteraria. La didattizzazione usa qui il "testo come pretesto" di riflessione sulla lingua e come strumento per la memorizzazione e l'automatizzazione di determinati elementi linguistici.

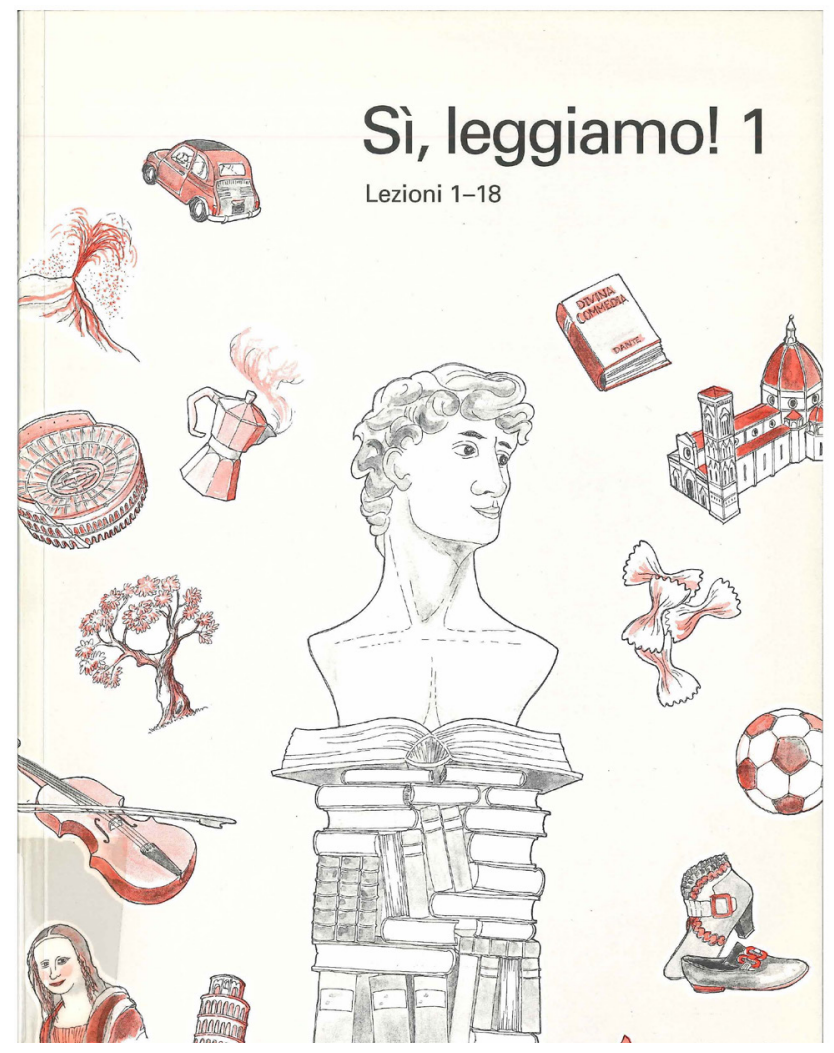

Fig. I: Il manuale Sì, leggiamo (Schärer Curetti 1994)

Con la diffusione di nuovi manuali orientati all'approccio comunicativo, l'insegnamento della lingua orientato al QCER si è più fortemente distinto dall'insegnamento della letteratura. Ecco allora che, se da una parte in determinate sequenze didattiche le letture forniscono innumerevoli spunti per creare situazioni comunicative autentiche, in altre fasi hanno primariamente l'obiettivo di avvicinare l'apprendente alla produzione letteraria della lingua target. 
Questo è l'approccio di Tra il dire e il fare (Jenny 2008; fig. 2), una raccolta di testi e dettagliati scenari didattici per leggere testi medievali in classi di liceo a partire dal livello BI. Jenny presenta diverse strategie sia per rendere accessibili testi che presentano grosse difficoltà linguistiche, sia per avvicinare l'apprendente alle tematiche e ai contesti storici dei testi scelti. Francesco d'Assisi viene così presentato come un ragazzo ribelle che potrebbe essere dei giorni nostri, e solo in un secondo momento, una volta creata quella attenzio-

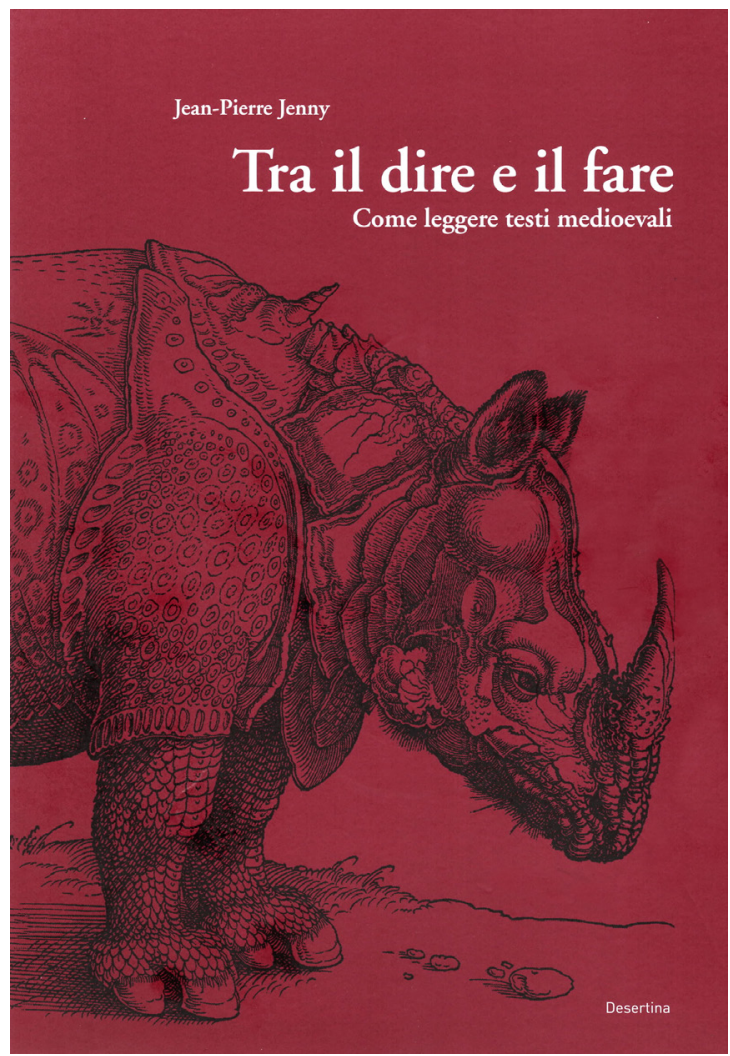

Fig. 2: Il manuale Tra il dire e il fare (Jenny 2008)

ne e prossimità che favoriscono un ascolto attivo, e approfondendo nozioni biografiche e storiche, il testo e l'autore sono situati nel contesto richiesto da un approccio analitico. In diversi corsi di didattica dell'italiano in Svizzera tedesca il volume viene raccomandato a studenti e studentesse nell'ottica di promuovere la loro competenza nell'adattare quei contenuti universitari a cui sono oramai abituati/e al pubblico di adolescenti liceali. 
Tre anni dopo, nel 20II, il Lehrmittelverlag di Zurigo pubblica una brossura dedicata agli insegnanti di letteratura straniera dal titolo Littura. Ideen für den Literaturunterricht (Pfau-Vetterli-Verstraete 20II; fig. 3). Il libro, improntato alla tradizione della didattica dialogica e con ciò molto vicino all'approccio ermeneutico, si intende come una raccolta di metodi per lavorare, prima, durante e dopo una lettura, su due aspetti centrali: la caratterizzazione dei personaggi e la struttura del testo. Molte delle attività proposte sollecitano reazioni personali del lettore a determinati elementi testuali, con lo scopo di riconoscere, approfondire e indagare rapporti intra- e intertestuali. Non raramente le attività proposte fanno ricorso a metafore (colori, alberi, emoticon, diagrammi) per sollecitare attività di analisi e interpretazione, o le sfruttano per costruire, insieme all'apprendente, quel vocabolario (presente in LI ma ancora da consolidare in L2/LS) necessario a indagare su personaggi e strutture testuali. In accordo con la didattica dialogica alla quale fanno riferimento le autrici, ogni attività prevede

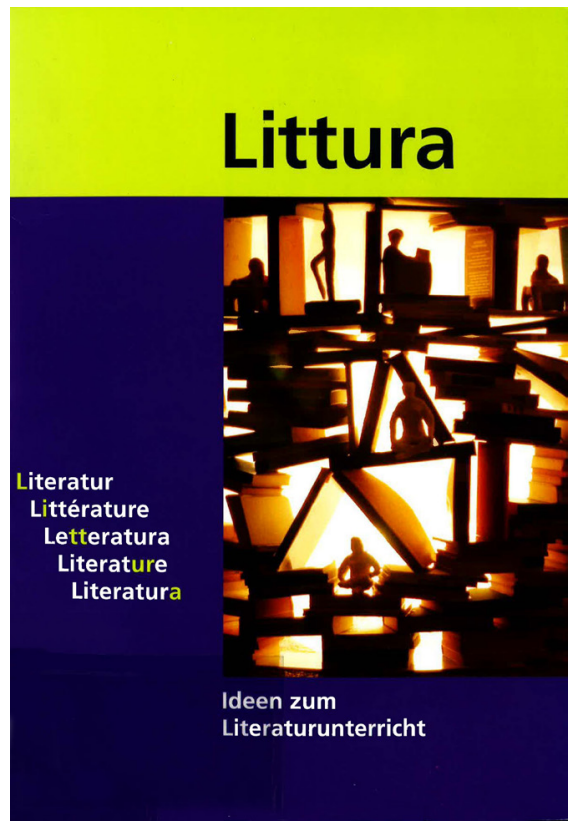

Fig. 3: Il manuale Littura (Pfau e Vetterli-Verstraete 20II)

la valorizzazione, attraverso il feedback insegnante-apprendente o apprendente-apprendente, dell'apporto personale al processo di analisi del testo, vegliando a che tale apporto personale resti tuttavia aderente al testo stesso - anche se, temporaneamente, lo si abbandona per attivare e indagare invece il processo di ricezione individuale. 
Altri tre anni dopo, esce per mano di un insegnante di liceo il volume Letteratura facile (Sorba 20I4; fig. 4), un'antologia per apprendenti che raccoglie 24 unità didattiche. Il volume rispecchia il bisogno di disporre di un manuale in cui «le nozioni fossero ridotte all'essenziale e il peso maggiore poggiasse sulla pratica: la Letteratura come qualcosa di vivo, presente, da poter capire subito, apprezzare e, nei limiti del possibile, persino imitare e riprodurre» (nota dell'autore). Il volume segue l'approccio tradizionale storico biografico combinato a un approccio stilistico-linguistico e privilegia una didattizzazione volta a focalizzare l'attenzione dell'apprendente sul contesto culturale e storico in cui l'opera è nata. Laddove necessario,

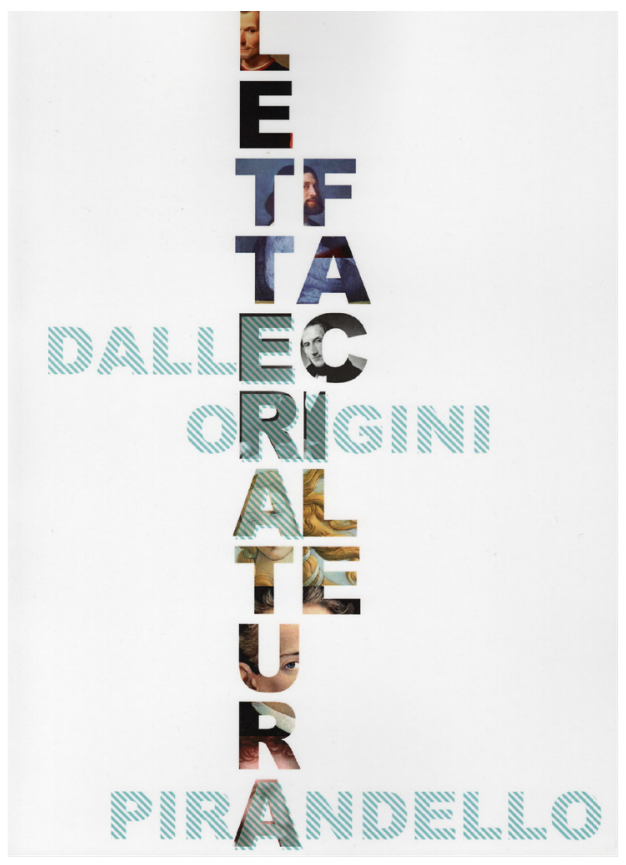

Fig. 4: Il manuale Letteratura facile (Sorba 20I4)

fornisce puntualmente gli strumenti e il lessico necessario all'analisi, nonché le nozioni indispensabili alla contestualizzazione. Le attività didattiche, organizzate in attività di pre-, while- e post-reading, mirano alla comprensione del testo e all'acquisizione di strumenti per l'analisi letteraria, fornendo all'apprendente piste predefinite dall'insegnante. Le fasi di interpretazione e i "momenti creativi" (p. es. l'invito a scrivere un sonetto) si situano generalmente a margine delle fasi di post-reading. 
Nel 2018 viene infine pubblicato il "portfolio della letteratura" svizzero (Portfolio 20I8) - un figlio del suo tempo, visto che esce quasi contemporaneamente al Companion Volume al QCER (Council of Europe 20I8), dove l'analisi e la critica del testo letterario sono inseriti tra le competenze di mediazione di testi creativi ${ }^{4}$. Pur non rientrando nella categoria della manualistica, il portfolio di letteratura e i suoi descrittori si rivelano improntati a una didattica che combina elementi tradizionali con approcci più recenti come la didattica dialogica e l'approccio ermeneutico. Il portfolio della letteratura elvetico potrebbe anch'esso avere un influsso sullo sviluppo della didattica della letteratura, intendendosi come uno strumento per la pianificazione didattica dell'insegnante e per la riflessione dell'apprendente - tuttavia, essendo stato pubblicato in una sezione accessibile solo tramite una password del PEL III, è finora passato largamente inosservato.

\section{Condivisione online}

I tre volumi di Jenny, Pfau e Vetterli-Verstraete e Sorba sono il frutto del desiderio di condividere con colleghi e colleghe i propri materiali didatticisono gli autori stessi che, concepito il loro manuale, hanno cercato una casa editrice che li pubblicasse. Il fatto che ultimamente non sia uscito alcun nuovo manuale potrebbe far pensare a un orientamento verso pubblicazioni extranazionali. Va da sé che gli e le insegnanti dei licei svizzero-tedeschi ne abbiano sempre fatto uso - primo fra tutti il volume Letteratura italiana per stranieri di Balboni e Biguzzi (2009). Il fenomeno che tuttavia potrebbe spiegare un'apparente improduttività manualistica e che più sta rivoluzionando il panorama editoriale di risorse per la didattica della letteratura italiana LS è legato a una peculiarità associazionistica elvetica. Gli insegnanti di italiano dispongono infatti già dal 2007 di un intranet nazionale integrato nella piattaforma collaborativa italianoascuola.ch (Alloatti-Ferrari 2007; fig. 5): dapprima un semplice dominio online e dal 2016 un'associazione sostenuta dall'Ufficio federale della Cultura con una piattaforma dalle funzionalità collaborative avanzate, italianoascuola.ch promuove lo scambio di informa-

4 I descrittori del portfolio svizzero non distinguono, come il Companion Volume, tra "Expressing a personal response" e "analysis and criticism" e non sono categorizzati per livelli AI-C2. Si tratta piuttosto di descrittori di opzioni ricettive (che forniscono i criteri per selezionare e caratterizzare i testi letterari) e di opzioni produttive (con spunti per guidare l'azione dell'apprendente in un contesto task-based): i 62 descrittori che ne risultano sono ordinati nelle categorie "testo", "testo-contenuto", "testo-lingua", "testo-struttura" e "testo-testo" e descrivono competenze di savoir, savoir faire e savoir être da sviluppare nell'incontro con il testo letterario. 


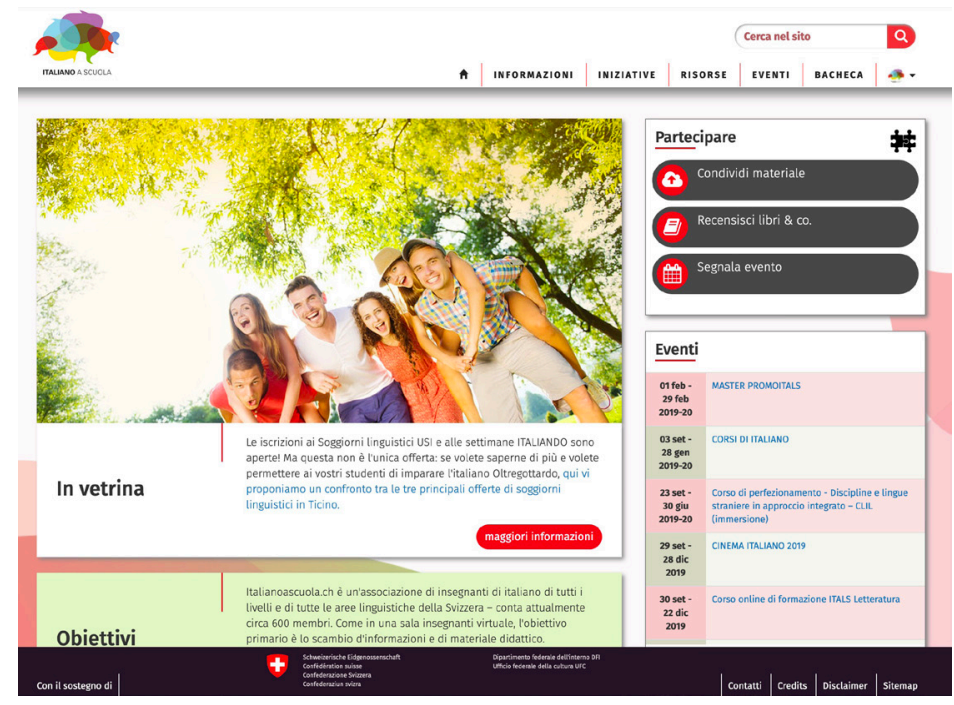

Fig. 5: La piattaforma collaborativa italianoascuola.ch

zioni e materiali didattici tra insegnanti di italiano sul territorio elvetico. Mentre nei primi anni di attività le risorse didattiche di natura letteraria si limitavano pressoché esclusivamente a liste di letture di classe e individuali (commentate), a esami di maturità sviluppati partendo da un testo letterario o a raccolte di testi di diversi generi (tra cui letterari) intorno a uno stesso tema, con l'introduzione della nuova piattaforma nel 2016 si è registrato un aumento sia del numero di utenti-autori/autrici che del numero di pubblicazioni di dossier di lettura e letteratura. Si tratta principalmente di fascicoli stampabili per l'apprendente che accompagnano nel dettaglio la lettura di una singola opera letteraria (dal racconto al romanzo, per una durata variabile da una breve sequenza a più mesi). Le attività variano notevolmente, prevedono in parte attività differenziate per interesse dell'apprendente, entrano nel dettaglio della comprensione, dell'analisi e dell'interpretazione dei passaggi più significativi dell'opera. A seconda dell'autore o dell'autrice del dossier, prevale un approccio tradizionale o ermeneutico, con un numero variabile di attività di natura interdisciplinare. Le tipologie di attività e i metodi seguiti sono variegati sia per approccio che per obiettivi principali delle attività. Alcune si limitano al livello della comprensione e dell'analisi, altre portano l'apprendente a interpretare, estrapolare, confrontare, o trasporre il testo oppure a reagire ad esso (cfr. categorie di attività orientate all'azione in Puren 2006). Il fatto che i dossier siano preconfezionati rappresenta sì un limite per lo sviluppo dei tipicamente imprevedibili percorsi di lettura, ma non impedisce che chi li costruisce sappia progettare, anche 
graficamente, gli spazi necessari a seguire inaspettate piste. Notevole è poi anche l'attività di pubblicazione di tali dossier da parte di insegnanti con pochi anni d'esperienza - la cultura della condivisione di documenti "fatti in casa", in cui l'errore può insinuarsi senza precludere una positiva ricezione del materiale, sembra farsi strada nelle generazioni più giovani e inibisce invece ancora fortemente le altre - eppure sono proprio anche quelle generazioni a servirsi dei dossier pubblicati -, l'alto numero di download sembra perlomeno indicarlo. E sono proprio questi dati che ci portano a immaginare che nuove tendenze nell'approccio al testo letterario possano prendere piede grazie all'innovazione dei singoli autori e delle singole autrici e che le loro "ricette", se dimostratesi nella pratica utili e accettate, si possano diffondere nuovi approcci con una velocità ben maggiore rispetto a quella tradizionale. Una produzione manualistica modulare (in dossier, per esempio), decentralizzata (con insegnanti contribuenti su tutto il territorio nazionale), autogestita e autoregolata, nonché vagliata nella sua qualità in tempi brevi si rivela essere uno scenario molto vicino a quello ipotizzato con "SwissEdupedia" nella pubblicazione Lehrmittel in einer digitalen Welt (Döbeli Honegger et al. 2018: I20).

\section{Letture privilegiate}

I criteri con cui l'insegnante sceglie il testo da trattare sono molteplici e in parte individuali (come non considerare che il testo proposto a una classe è, perlopiù e in virtù della Lehrfreiheit, un testo amato dall'insegnante?). I criteri più generalizzabili sono:

I. difficoltà linguistica: la complessità linguistica è adatta alla classe?

2. difficoltà nel lavoro di analisi: la classe dispone delle capacità cognitive che l'analisi e l'interpretazione del testo richiedono?

3. potenziale culturale: in quale misura il testo permette di avvicinare l'apprendente al mondo italofono, di sviluppare in lui comprensione, empatia, tolleranza all'ambiguità? In quale misura gli impliciti culturali presenti possono sollecitare curiosità e riflessioni o invece ostacolare la comprensione del testo?

4. tematica: i temi del testo possono interessare degli adolescenti svizzero-tedeschi?

5. estensione: la lunghezza del testo permette di trattarlo in un tempo ragionevole?

6. tutti i criteri suddetti contemporaneamente: un testo può essere adatto solo a uno/a o più apprendenti e quindi essere consigliato come lettura individuale?

La distinzione "lettura di classe" e "lettura individuale" ha confini meno netti di quanto possa sembrare. Generalmente le letture individuali sono 
oggetto di attività e test individualizzati nel corso degli ultimi anni di liceo e sono onnipresenti nelle liste di lettura per l'esame orale di maturità. Ma non sono rari i contesti in cui la lettura individuale si svolge all'interno di un circolo di lettura di impronta costruttivista (Alloatti-Palm 20IO: IO-II) o in scenari task-based in cui l'expertise che il singolo acquisisce contribuisce a moltiplicare saperi e competenze su un ambito più vasto (per esempio una corrente letteraria, un autore o un'autrice, un ambito tematico). Le modalità con cui un insegnante assegna all'apprendente una lettura individuale possono essere diverse: alcuni concedono la più ampia libertà di scelta (col rischio di imbattersi in un testo di bassa qualità letteraria o con la consapevolezza che la scelta può vertere su un genere non letterario), altri propongono alle classi delle "liste di letture personali". Su italianoascuola.ch se ne trovano diverse, tra cui alcune tematiche e commentate in dettaglio e altre cronologiche con indicazioni di difficoltà dei testi - tutte propongono letture integrali.

Per quanto riguarda le letture di classe, non è nota la proporzione tra unità didattiche di letteratura che seguono raccolte antologiche o una selezione di testi integrali. L'unico "rilevamento" sull'uso di testi letterari a scuola è stato pubblicato da Babylonia e compilato dall'oramai ultima libreria in Svizzera tedesca specializzata in lingue romanze e il loro insegnamento, la Libromania di Berna. Tra i I6 volumi più venduti (Stocker 20I6; fig. 6) notiamo, oltre a quattro letture per stranieri della Cideb, una prevalenza di testi degli ultimi decenni, due soli classici (La locandiera di Goldoni e Una storia semplice di Sciascia), una sola opera elvetica (Il giudice e la rondine di Fazioli) e l'assenza di letteratura femminile. Possiamo presumere che questa lista dei "più venduti" contempli le cosiddette "letture di classe", quindi volumi ordinati in gran numero dall'insegnante per le sue classi. Notiamo inoltre che le raccolte di racconti sono assenti - probabilmente perché i testi brevi possono essere fotocopiati.

La lista rispecchia l'ampio spettro di competenze linguistiche (da AI a B2) con cui l'insegnante di italiano deve fare i conti al liceo. Destinati ai livelli più bassi sono per esempio le letture semplificate della Cideb. Tra i primi testi autentici che si possono leggere integralmente anche già a fine A2/inizio BI c'è il brevissimo Un bacio di Ivan Cotroneo. In crescendo per complessità linguistica si trova poi Febbre gialla di Lucarelli o Per questo mi chiamo Giovanni di Garlando. Un altro livello di difficoltà che deve essere valutato dall'insegnante è quello relativo ai processi cognitivi che l'attività didattica richiede: il primo posto nella classifica dei più venduti, Nel mare ci sono $i$ coccodrilli di Geda, è per esempio un testo che può essere compreso anche solo a livello referenziale. Al sesto posto troviamo invece la sceneggiatura del film La vita è bella - un'opera che reclama, per essere trattata, no- 


\begin{tabular}{|c|c|c|c|}
\hline & Autore & Titolo & Casa editrice \\
\hline 1 & Geda, Fabio & Nel mare ci sono i coccodrilli & Reclam \\
\hline 2 & Goldoni, Carlo & La locandiera & Bonacci \\
\hline 3 & Ammaniti, Niccolò & lo e te & Reclam \\
\hline 4 & & La ricetta segreta & Cideb \\
\hline 5 & & Giallo al Crand Hotel du Lac & Cideb \\
\hline 6 & Benigni, Roberto & La vita è bella & Reclam \\
\hline 7 & Lucarelli, Carlo & Febbre gialla & EL \\
\hline 8 & Cotroneo, Ivan & Un bacio & Bompiani \\
\hline 9 & Garlando, Luigi & Per questo mi chiamo Giovanni & BUR extra \\
\hline 10 & Fazioli, Andrea & Il giudice e la rondine & Guanda \\
\hline 11 & Baricco, Alessandro & Novecento & Feltrinelli \\
\hline 12 & Carofiglio, Gianrico & Testimone inconsapevole & Sellerio \\
\hline 13 & Baricco, Alessandro & Seta & Feltrinelli \\
\hline 14 & Manzoni, Alessandro & I promessi sposi (livre et cd) & Cideb \\
\hline 15 & Folco, Daniela & Baci da Venezia & Cideb \\
\hline 16 & Sciascia, Leonardo & Una storia semplice & Adelphi \\
\hline
\end{tabular}

Fig. 6: Libri più venduti a Libromania (Stocker 2016)

tevoli capacità di analisi e interpretazione. Lo stesso vale per i ben due testi di Baricco, Novecento e Seta, oramai dei "classici" nei licei svizzero-tedeschi.

La lista di Libromania, oltre a non essere più attuale (si riferisce al 20I6), non può tuttavia essere considerata rappresentativa. Probabilmente la maggioranza degli e delle insegnanti acquista volumi da altre fonti (librerie in Svizzera italiana o in Italia e grandi distributori online).

Stando tuttavia alle opere letterarie recensite e ai materiali didattici pubblicati su italianoascuola.ch, nelle classi di liceo della Svizzera tedesca si leggono anche (a titolo esemplificativo, in ordine alfabetico d'autore) $R e-$ sto qui e L'ultimo arrivato di Balzano, Niente, più niente al mondo di Carlotto, Non dirmi che hai paura di Catozzella, Bianca come il latte, rossa come il sangue di D'Avenia, L'Arminuta di Di Pietrantonio, Amalia di Garberoglio, Fulmine di Gurrado, Scontro di civiltà per un ascensore in Piazza Vittorio di Lakhous, La misura dell'uomo di Malvaldi, Mio fratello rincorre i dinosauri di Mazzariol, Sostiene Pereira di Tabucchi o Cacciateli! di Vecchio. Sia detto a margine che in Svizzera francese le letture possono presentare difficoltà linguistiche ben maggiori (Il fotografo di Auschwitz di Crippa o Tra due mari di Abate).

Tra le graphic novel si annoverano pochi titoli, tra cui Dove non sei tu di Lorenzo Ghetti, Macaroni! di Campi e Zabus e Il grande ghetto di Nardella e Bizzarri - forse un indizio del fatto che il genere sta solo iniziando a essere considerato un candidato per la lettura a carattere letterario. Infine, dalle 
informazioni tratte dalle recensioni e dalle risorse pubblicate su italianoascuola.ch, risultano letti spesso dei testi brevi di Benni, Buzzati, Calvino, Campana, Rodari, Romagnoli, Pirandello, Sciascia e Verga, nonché testi brevi compresi nelle raccolte rosse con note di vocabolario della casa editrice Reclam ${ }^{5}$ - un formato privilegiato nelle classi sia per racconti che per romanzi visto il basso costo dei volumi e la presenza di note di vocabolario.

\section{Conclusione}

Indipendentemente dagli approcci didattici seguiti nelle singole classi dei licei della Svizzera tedesca, concludiamo con delle ipotesi di sviluppi nei prossimi anni.

Consci del fatto che l'approccio comunicativo ha co-determinato scelte didattiche a vantaggio di testi d'attualità, argomentativi e pseudo-dialogici che hanno in parte portato certi e certe insegnanti a ridurre il numero di ore dedicate alla letteratura, si ipotizza in questa sede una controtendenza. 亡̀ immaginabile che il dibattito politico-educativo verta presto sulla rilevanza dell'insegnamento linguistico in un'epoca in cui i traduttori automatici ci permetteranno di esprimerci anche in lingue sconosciute. Ma è proprio in questo ambito che la letteratura al liceo può giustificare un mantenimento della dotazione oraria della materia "italiano" in tempi non troppo lontani, in cui sia l'ORM che il piano quadro dovranno essere rivisti - e diverse nuove materie tra cui l'informatica entreranno a far parte dei programmi scolastici. La riforma privilegerà, e questo orientamento è già in atto, discipline e contenuti in grado di sviluppare le competenze del xxi secolo, ossia le competenze non automatizzabili, tra cui il pensiero critico, la creatività, la comunicazione e le competenze sociali. Tutte queste competenze sono al contempo premessa e obiettivo di un insegnamento della letteratura sia in chiave stilistico-linguistica che ermeneutica o orientata all'azione (e ancor più nella combinazione di questi approcci). Sono infatti i testi letterari che, in maniera privilegiata, permettono di allenare l'apprendente alla polisemia dei messaggi testuali. Essi sviluppano la capacità di comprensione inferenziale, di analisi degli elementi retorici che sono chiavi di lettura interpre-

5 Tra le raccolte di testi brevi della collana: Racconti italiani del xx secolo, Pecore nere, Amori bicolori, Il bar sotto al mare di Benni, I5 novelle de Il Decameron di Boccaccio e le Favole al telefono di Rodari. Altri titolo della collana: Io e te e Io non ho paura di Ammaniti, Mal di pietre di Agus, e Seta di Baricco, Palomar di Calvino, Volevo i pantaloni di Cardella, Le avventure di Pinocchio di Collodi, L'Arminuta di Di Pietrantonio, Per questo mi chiamo Giovanni di Garlando, Nel mare ci sono i coccodrilli di Geda, I promessi sposi di Manzoni, Sei personaggi in cerca d'autore di Pirandello, Sostiene Pereira di Tabucchi, Va' dove ti porta il cuore di Tamaro. Particolarmente adatta al livello liceale della Svizzera tedesca è anche l'antologia "Letteratura italiana: da Francesco d'Assisi a Paolo Giordano". 
tative dei messaggi che veicolano. La letteratura è una palestra di autentico ascolto, introspezione e interpretazione di sé, dell'altro e del mondo che può portare a quell'empatia necessaria affinché si raggiunga vera comunicazione di messaggi - messaggi che non si definiscono solo dai fatti che rapportano, ma dalla soggettività che veicolano attraverso percezioni e intenzioni, o (facendo riferimento al modello delle quattro orecchie di Schulz von Thun) dalle relazioni, dagli appelli e dalle rivelazioni di sé. L'esercizio di ascolto è poi tanto più efficace quando avviene in una lingua non prima, superando quindi le difficoltà implicite nel contatto con un'altra lingua-cultura e rendendosi capaci di dimostrare una competenza di ascolto interculturale. Ecco allora che risultano evidenti i limiti dei traduttori automatici, che tutt'al più possono aiutarci a comprendere messaggi referenziali. Le difficoltà nel cogliere la polisemia di un testo (anche non letterario) si manifestano palesi infatti appena si prova a tradurre letteratura. Ma la traduzione automatica non è l'unico sviluppo tecnologico in grado di influire sulle modalità di insegnamento della letteratura in lingua straniera: la sfida per l'insegnante di italiano nel prossimo futuro risiede anche nella sua capacità di cogliere i mutamenti della digitalizzazione sulla sua disciplina e sulla produzione letteraria - pensiamo ai fenomeni di scrittura collettiva o alla sempre meno netta distinzione di generi testuali e multimediali. In tal senso si muove anche il Portfolio di letteratura svizzero, che considera "letteratura" un'ampia gamma di produzioni artistiche, tra cui i romanzi grafici e le produzioni filmiche. E non da ultimo, rappresenterà una sfida anche il saper integrare le possibilità offerte dalle nuove tecnologie a livello didattico: da alcuni anni sono richiestissimi corsi di formazione per insegnanti sull'integrazione delle tecnologie digitali in classe e recentemente si è vagliata la possibilità di rendere accessibili anche ai e alle liceali dei corsi universitari elearning di letteratura. Un'inversione verso una didattica accademica? Non credo: è nella combinazione di approcci diversi che si possono sfruttare al meglio le grandi potenzialità didattiche offerte dal testo letterario.

\section{Bibliografia}

AA. VV. Portfolio di letteratura: elemento aggiuntivo al PEL III per l'insegnamento della letteratura nei licei, (documenti scaricabili nell'intranet), Berna, Schulverlag Plus, 20I8, www.portfoliodellelingue.ch.

Alloatti, Sara - Ferrari, Reto, Italianoascuola.ch: la "sala insegnanti virtuale" di chi insegna italiano in Svizzera, dal 2007 al 2019 su italianoascuola.ch, Zürich-Beromünster, online dal 2007.

Alloatti, Sara - Palm, Pascale, SOL-Szenarien für den Französisch- und Italienischunterricht. Zürich, dispensa per corsi di formazione continua dell'Università di Zurigo, 20 Io. 
Balboni, Paolo Ernesto - Biguzzi, Anna, Letteratura italiana per stranieri, Perugia, Guerra, 2009.

Council of Europe, Common European Framework of reference for languages: learning, teaching, assessment. Companion volume with new descriptors, 2018, www.coe.int/lang-cefr.

Döbeli Honegger, Beat - Hielscher, Michael - Hartmann, Werner, Lehrmittel in einer digitalen Welt. Expertenbericht im Auftrag der Interkantonalen Lehrmittelzentrale (ilz), 20I8, www.ilz.ch.

Jenny, Jean-Pierre, Tra il dire e il fare: come leggere testi medioevali, Chur, Desertina, 2008.

Magnani, Mirco, «Il testo letterario e l'insegnamento delle lingue straniere», Studi di Glottodidattica, 2009, I, pp. I07-II3.

Pfau, Anita - Vetterli-Verstraete, Martine, Littura. Ideen für den Literaturunterricht, Zürich, Lehrmittelverlag des Kantons Zürich, 20II.

Puren, Christian, Explication de textes et perspective actionnelle: la littérature entre le dire scolaire et le faire social, Association française des Professeurs Français de Langues Vivantes, 2006, www.aplv-languesmodernes.org/ spip.php?article389.

Schärer Curetti, Elettra, Sì, leggiamo!, Zurigo, Lehrmittelverlag des Kantons Zürich, I994.

Sorba, Manlio, 24 unità didattiche di letteratura facile dalle origini a Pirandello, per studenti d'italiano L2 (livello B2-CI), Winterthur, edizione propria, 2014 .

Stagi Scarpa, Mariella (a cura di), Insegnare letteratura in lingua straniera, Roma, Carrocci, 2005.

Stocker, Letizia, «Letture in classe», Babylonia, 3, 2016, pp. IO2-IO3. 
